\title{
Prognostic Factors and Recurrence Pattern of Far-advanced Gastric Cancer with Pathologically- positive Para-aortic Lymph Nodes
}

\author{
AKIO KAITO $^{1,2}$, TAKAHIRO KINOSHITA ${ }^{1}$, MASANORI TOKUNAGA ${ }^{1}$, \\ HIDEKI SUNAGAWA ${ }^{1}$, MASAHIRO WATANABE ${ }^{1}$, SHIZUKI SUGITA ${ }^{1}$,

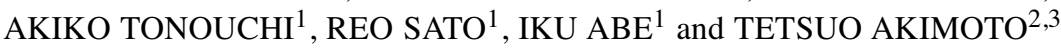 \\ ${ }^{1}$ Gastric Surgery Division, National Cancer Center Hospital East, Kashiwa, Japan; \\ ${ }^{2} J u n t e n d o$ University Graduate School of Medicine, Tokyo, Japan; \\ ${ }^{3}$ Department of Radiation Oncology, National Cancer Center Hospital East, Kashiwa, Japan
}

\begin{abstract}
Background: The role of para-aortic lymph node (PALN) dissection for far-advanced gastric cancer is controversial in patients with clinical PALN positivity who have responded to chemotherapy. Materials and Methods: We retrospectively analyzed long-term outcomes of patients with pathologically-positive PALNs who underwent radical gastrectomy. Results: The 3- and 5-year overall survival (OS) rates of 65 pathologically $P A L N$-positive patients who underwent PALN dissection ( $n=704$ ) were $33.8 \%$ and $21.2 \%$, respectively. Multivariable analysis revealed the following poor prognostic factors: nodal involvement around the celiac axis (hazard ratio $(H R)=4.04,95 \%$ confidence interval $(C I)=1.55$ 9.63), tumor diameter of $\geq 120 \mathrm{~mm}$ ( $H R=3.37$; $95 \% \mathrm{CI}=1.18$ 9.63) and $\geq 3$ PALNs involved (HR=2.24; 95\% CI=1.21-4.15). Patients with none of these factors survived significantly longer than those with any of these factors (5-year OS $=87.5 \%$ versus $9.3 \%$, respectively; $p<0.001)$. Conclusion: Pathologically $P A L N$-positive patients achieve long survival; however, the indications for PALN dissection should be carefully considered.
\end{abstract}

Para-aortic lymph node (PALN) metastasis from gastric cancer is classified as distant metastasis in both the 7th classification of the International Union against Cancer (1) and the 3rd English edition of the Japanese Gastric Cancer

This article is freely accessible online.

Correspondence to: Takahiro Kinoshita, MD, PhD, FACS, Gastric Surgery Division, National Cancer Center Hospital East, 6-5-1 Kashiwanoha, Kashiwa, Chiba 277-8577, Japan. Tel: +81 471331111, Fax: +81 471309960, e-mail: takkinos@east.ncc.go.jp

Key Words: Stomach neoplasms, para-aortic lymph node, extended lymphadenectomy, locoregional neoplasm recurrence, prognosis.
Classification (2). Thus, systemic chemotherapy is recommended as first-choice treatment in the current guidelines (3). In patients with advanced gastric cancer, the reported incidence of pathological metastasis to the PALNs is $8.5-30.0 \%(4-8)$. In one study, micrometastasis was detected by immunohistochemical staining in 64\% of patients who underwent prophylactic PALN dissection (9). In several Japanese specialized centers, including ours, prophylactic PALN dissection was aggressively performed for advanced gastric cancer from the 1980s to the early 1990s. In 1995, a multi-institutional prospective randomized controlled trial comparing standard D2 dissection versus D2 plus PALN dissection for serosa-positive advanced gastric cancer without gross metastasis to the PALNs was conducted in Japan (JCOG9501) (10). This trial proved that the 5-year overall survival (OS) rates did not differ between the two groups $(69.2 \%$ vs. $70.3 \%$, respectively) and concluded that prophylactic PALN dissection is not effective (6). However, the incidence of pathologically positive PALNs in this trial was only $8.5 \%$; thus, the benefit of PALN dissection for those patients could not be fully investigated.

In general, the prognosis of patients with pathologicallypositive PALNs is thought to be unfavorable, even after systematic PALN dissection. However, it is known that a certain subpopulation of these patients can achieve long survival. The reported postoperative 5-year survival rate of patients with pathologically-positive PALNs is $16-25 \%(4,6$, $8,11,12)$. Due to progress in modern powerful perioperative chemotherapy regimens, it was recently suggested that the prognosis of these patients can be improved. A recent phase II trial (JCOG 0405) investigated the feasibility of neoadjuvant chemotherapy (2-3 cycles of S1+cisplatin) followed by radical gastrectomy with systematic PALN dissection for patients with clinically diagnosed metastasis to the PALNs (13). The R0 resection rate was $82 \%$ and the 5 -year OS rate $57 \%$. These 
encouraging results imply that such multimodal treatment is promising for patients with clinically positive PALNs (3). Conversely, a Chinese clinical trial of preoperative administration of capecitabine and oxaliplatin followed by radical gastrectomy without PALN dissection for patients with PALN involvement suggested that a survival benefit could only be obtained in responders to chemotherapy who could undergo R0 resection (14). Meanwhile, the reported median survival time of patients with clinically positive PALNs who underwent only chemotherapy without surgery is 9.8 to 12.8 months $(15,16)$. Given this evidences we presume that a multimodal treatment strategy may provide a survival benefit for selected patients, most likely responders to chemotherapy.

In this context, identification of prognostic factors for patients with PALN metastasis seems important; however, it has not been well investigated to date. Previous studies have indicated as prognostic factors for these patients the macroscopic type, overall number of involved nodes (17), number of PALN metastases (4), patient's age and PALN metastatic site (station No. a2/b1, according to Japanese guidelines) (8). However, most of these studies included few patients and did not investigate the recurrence site. In this retrospective study, we investigated prognostic factors, longterm outcomes, as well as recurrence status, using the medical database of one high-volume cancer center in Japan.

\section{Patients and Methods}

Patients who underwent radical gastrectomy with PALN dissection for advanced gastric cancer from June 1992 to December 2014 in our Institution were included in this study. The clinicopathological features, survival, recurrence pattern and prognostic factors of patients with pathologically identified PALN involvement after curative gastrectomy were analyzed. Patients who could not undergo curative resection were excluded. Curative resection was defined as R0 gastrectomy without evidence of a residual tumor as described in the Japanese Classification of Gastric Carcinoma, 3rd edition (2).

The primary end-point of the present study was detection of prognostic factors in patients undergoing radical gastrectomy with pathologically proven PALN involvement. The secondary end-point was investigation of the association between PALN involvement and recurrence pattern.

This study was approved by the Institutional Review Board of the National Cancer Center, Japan (IRB file No. 2016-299; approval date: January 12, 2017).

Surgical procedures and postoperative follow-up. The patients included in this study underwent standard gastrectomy with D2 lymphadenectomy and complete or incomplete PALN dissection without an evident residual tumor. D2 lymphadenectomy is described in the Japanese gastric cancer treatment guidelines (ver. 4) (3). Splenectomy had been performed for all patients with advanced gastric cancer who underwent total gastrectomy before 2009; patients without cancer invasion to the greater curvature of the upper third of the stomach did not undergo splenectomy after 2010. Complete PALN dissection was defined as removal of station
No. 16a2 (PALNs between the level of the celiac axis and left renal vein) and No. 16b1 (PALNs between the left renal vein and inferior mesenteric artery). However, in patients who underwent distal subtotal gastrectomy, dissection of the left upper lateral nodes (No. 16a2-lat) was optional (10).

Complete PALN dissection was prophylactically performed in patients with a clinically diagnosed tumor deeper than $\mathrm{T} 2$ before 2001, when patients' enrollment in the JCOG 9501 trial was complete. After 2002, complete PALN dissection was performed only for patients with macroscopically confirmed PALN involvement that could be resected without an evident residual tumor. Neoadjuvant chemotherapy was performed after 2005, after enrollment in the JCOG 0405 trial had started (13). Postoperatively, S-1 monotherapy was administrated as adjuvant chemotherapy starting in 2001, after enrollment in the ACTS-GC trial had started in Japan (18).

Outpatient follow-up involved physical examination, blood tests, including tumor marker evaluation, and chest/abdominal computed tomography scans every 3 months for the first 2 years and then every 6 months until, at least, 5 years postoperatively.

Data collection and analysis. The clinicopathological data of the patients included in this study were collected from the electronic patients' database of our Division. The clinical records of patients with pathologically proven PALN positivity were reviewed and additional information was collected (type of surgical procedure, including the extent of PALN dissection; existence and site of tumor recurrence; and survival).

Tumor staging followed the 7th classification of the International Union against Cancer (1) and the definitions of macroscopic subtypes, histological subtypes, as well as lymph node stations (including the PALNs), followed the 3rd English edition of the Japanese Gastric Cancer Classification (2). Grading of postoperative complications was performed according to the Clavien-Dindo criteria (19). Intra-abdominal infectious complications included anastomotic leakage, pancreatic fistula and intra-abdominal abscess (either alone or concurrently).

In this study, histologically confirmed tubular and papillary adenocarcinomas were defined as differentiated adenocarcinomas, while poorly differentiated adenocarcinomas and signet-ring cell carcinomas were defined as undifferentiated adenocarcinomas.

Statistical analysis. All continuous variables are presented as median (range). Kaplan-Meier analysis and log-rank tests were used to assess associated factors, while multivariate Cox proportionalhazard analysis was conducted to detect independent prognostic factors in patients with PALN involvement. All statistical analyses were performed using SPSS Statistics 20 (IBM Corp., Armonk, NY, USA) and $p<0.05$ was considered statistically significant.

\section{Results}

In total, 704 patients underwent gastrectomy with PALN dissection, with 107 patients $(15.2 \%)$ being pathologically diagnosed with positive PALNs. Of these 107 patients, 4 who underwent simultaneous resection of other cancers and 38 who had non-curative factors other than resectable macroscopic PALN metastasis were excluded from this study. Finally, 65 patients who underwent curative 
gastrectomy with PALN dissection were included in this study as shown in Figure 1.

The non-curative factors of the excluded patients were unresectable lymph node metastasis in 16 patients, peritoneal metastasis in 5 , positive washing cytology in 8 , unresectable positive resection margin in 3 and of unknown reason based on the information from our database or surgical records in 6.

Patients' background characteristics. The background characteristics of the patients included in this study are shown in Table I. Macroscopic type 4 tumors were observed in $12(18 \%)$ patients. Total gastrectomy with splenectomy was performed in $48(74 \%)$, combined resection of other organs was performed in $16(25 \%)$, whereas a thoracoabdominal approach was performed in 7 . Complete PALN dissection (No. 16a2/b1) was performed in 32 patients (49\%). Neoadjuvant and adjuvant chemotherapy were performed in $10(15 \%)$ and $23(35 \%)$ patients, respectively. Postoperative intra-abdominal complications (grade $\geq$ IIIa) occurred in 13 (20\%) patients with no in-hospital deaths. The median number of pathologically-positive PALNs was 2 and the site of PALN metastasis was a2 in $16(25 \%)$ patients, b1 in $24(37 \%)$ and both $\mathrm{a} 2$ and b1 in $25(38 \%)$.

Survival analysis. The median follow-up time was 19.7 months (range $=2.8-143.7$ ). The 3- and 5-year OS rates were $33.8 \%$ and $21.2 \%$, respectively, with a median survival time of 20.3 months. Kaplan-Meier analysis was conducted to investigate prognostic factors in patients with pathologically positive PALNs (Table II). Patients with macroscopic type 4 tumors, a tumor diameter of $\geq 120 \mathrm{~mm}$, positive nodes $\geq 10$, positive PALNs $\geq 3$ and PALN involvement in both a 2 and b1 nodes had significant poorer prognoses according to the log-rank test. The tumor location, histological subtypes, extent of PALN dissection, pathological tumor depth, as well as lymphatic invasion, had no significant effect on the prognosis of patients with pathologically-positive PALNs.

A comparison of the 5-year OS rate of patients with or without regional lymph node metastasis is shown in Figure 2. Patients with No. 8a, 9 and 11 nodal involvement had a significantly poorer prognosis than those without such nodal involvement, according to the log-rank test.

The prognostic factors for patients with PALN involvement were investigated using multivariate Cox regression analysis as shown in Table III. More than two involved PALNs (hazard ratio $(\mathrm{HR})=2.24 ; 95 \%$ confidence interval $(\mathrm{CI})=1.21-4.15)$, a tumor diameter of $\geq 120 \mathrm{~mm}$ $(\mathrm{HR}=3.37 ; 95 \% \mathrm{CI}=1.18-9.63)$ and nodal involvement of station No. 8a, 9 and/or $11(\mathrm{HR}=4.04 ; 95 \% \mathrm{CI}=1.55-10.5)$ were independent poor prognostic factors.

Kaplan-Meier analysis results of patients with or without these poor prognostic factors are shown in Figure 3. The 3and 5-year survival rates of patients with any of these

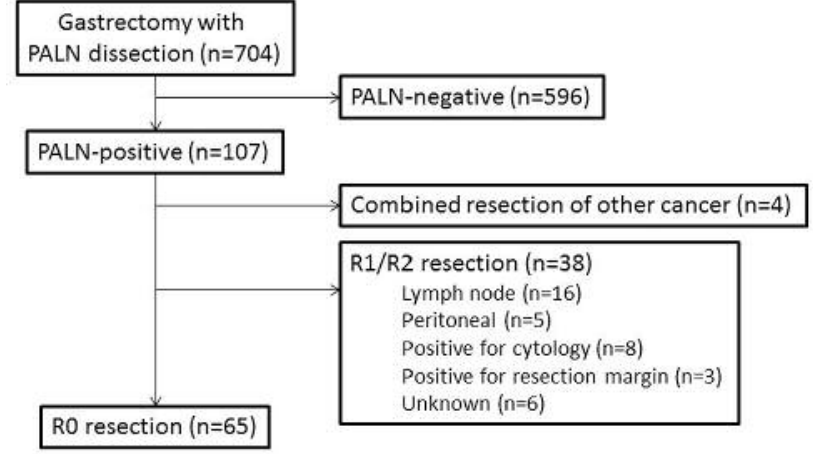

Figure 1. Disposition of the patients. Para-aortic nodal involvement was identified in 107 of 704 patients who underwent gastrectomy with paraaortic nodal dissection. Of these, 4 patients who had simultaneous resection of other cancer and 38 patients who had non-curative factor other than resectable macroscopic PALN metastasis were excluded from this study. In total, 65 patients who had curative gastrectomy with PALN dissection were included.

prognostic factors (tumor diameter of $\geq 120 \mathrm{~mm}, \geq 3$ involved PALNs and nodal involvement of No. 8a, 9 and/or 11) were $24.1 \%$ and $9.4 \%$ respectively, while those of patients without these factors were $87.5 \%$ (both 3- and 5-year OS rates). The OS of patients without these prognostic factors was significantly better than the OS of patients with any of these factors (log-rank $p<0.001)$.

Recurrence analysis. Postoperative tumor recurrence was observed in $49(75 \%)$ patients with a median recurrence-free survival duration of 11.6 months. The initial site of recurrence was lymph node in 24 patients, peritoneum in 15 , blood in 9 , bone in 3 and pleura in 2 (Table IV). Lymph node recurrences were observed in locoregional lymph nodes in 8 patients, distant lymph nodes in 1 and PALNs in 15 (a2 in 9 and b1 in 12 with duplication). In 10 patients, PALN recurrence was observed at the same site at which PALN dissection was performed during the initial gastrectomy, while the remaining 5 patient developed relapse at the site at which the PALNs had not been dissected during the initial gastrectomy.

\section{Discussion}

In the clinical setting, physicians must sometimes decide whether to excise the PALNs with the primary tumor after a patient with gastric cancer has responded to chemotherapy; such patients are considered to have limited metastatic gastric cancer. At present, only one ongoing randomized controlled study (FLOT 5) is assessing the role of surgery for such patients. The FLOT 5 study was designed to investigate the superiority of chemotherapy plus surgery compared to chemotherapy alone for patients with limited metastatic gastric 
Table I. Background characteristics of patients with pathologically-positive para-aortic lymph nodes.

\begin{tabular}{|c|c|c|c|}
\hline Characteristics & Value & Characteristics & Value \\
\hline Number of patients & 65 & No. of retrieved lymph nodes (median) & 85 \\
\hline Gender & & (range) & $(39-309)$ \\
\hline Male & 35 & No. of retrieved PALN (median) & 10 \\
\hline Female & 30 & (range) & $(1-52)$ \\
\hline Age (year, median) & 61 & PALN dissection & \\
\hline (range) & $(37-73)$ & Incomplete & 33 \\
\hline Tumor location & & Complete & 32 \\
\hline Upper third of stomach & 28 & Postoperative intra-abdominal complication* & 13 \\
\hline Middle third of stomach & 20 & Postoperative hospital stay (days, median) & 23 \\
\hline Lower third of stomach & 16 & (range) & $(8-115)$ \\
\hline Whole stomach & 1 & Adjuvant chemotherapy & \\
\hline Macroscopic type & & Presence & 23 \\
\hline 0 & 2 & Absence & 42 \\
\hline 1 & 1 & Follow-up periods (months, median) & 19.7 \\
\hline 2 & 9 & (range) & $(2.8-143.7)$ \\
\hline 3 & 36 & Histology & \\
\hline 4 & 12 & Differentiated & 16 \\
\hline 5 & 5 & Undifferentiated & 49 \\
\hline Neoadjuvant chemotherapy & & Pathological tumor depth & \\
\hline Presence & 10 & $\mathrm{~T} 1$ & 1 \\
\hline Absence & 65 & $\mathrm{~T} 2$ & 3 \\
\hline Operation procedure & & T3 & 13 \\
\hline Total gastrectomy & 48 & $\mathrm{~T} 4$ & 48 \\
\hline Distal subtotal gastrectomy & 16 & Tumor diameter (mm) & 80 \\
\hline Pancreaticoduodenectomy & 1 & (range) & $(15-200)$ \\
\hline Combined resection & & Overall No. of positive nodes (median) & 19 \\
\hline Splenectomy and distal pancreatectomy & 13 & (range) & $(1-126)$ \\
\hline Splenectomy & 35 & No. of positive PALN (median) & 2 \\
\hline Other & 14 & (range) & $(1-26)$ \\
\hline Approach & & Site of PALN metastasis & \\
\hline Abdominal & 58 & $\mathrm{a} 2$ & 16 \\
\hline \multirow[t]{2}{*}{ Thoracoabdominal } & 7 & b1 & 24 \\
\hline & & both (a2 and b1) & 25 \\
\hline
\end{tabular}

* $\geq$ Grade 3 according to Clavien-Dindo classification; PALN, para-aortic lymph node.

cancer or esophagogastric junction cancer (NCT02578368), including those with PALN metastasis. However, because no clear guidelines are currently available, physicians must make decisions with consideration of each patient's condition or disease status. In this sense, knowledge of specific prognostic factors for patients with pathologically positive PALNs using available data seems important to avoid futile surgical invasiveness because this disease situation is fundamentally unfavorable. Our Japanese high-volume center has a history of aggressive PALN resection. Therefore, we thought that the information in our database should be maximally utilized.

In the current study, three poor prognostic factors were identified. The number of positive PALNs has also been pointed out as a prognostic factor in previous studies $(4,20)$. As in patients with limited metastatic gastric cancer, the number of liver metastases (one or two) has been identified as a prognostic factor after surgical resection when no extrahepatic metastasis is present (21). It is presumed that the number of lesions might be a pivotal factor associated with the prognosis in such patients with limited metastasis. When the number of positive PALNs is one or two, metastatic nodes tend to be located in a limited area instead of being widespread. In the present study, the OS of patients with metastasis at a single site ( 22 or b1) was higher than that of patients with metastasis at both sites. These results suggest that indications for PALN dissection should be carefully considered in patients with a relatively large number of enlarged PALNs spreading to sites both above and below the left renal vein. In that respect, systematic PALN dissection might not have to be performed in the majority of cases.

Concurrent metastasis to nodes around the celiac artery (station Nos. 8a, 9 or 11) was also identified as a poor prognostic factor. Similarly, the results of the JCOG 0405 trial revealed that patients with both PALN and bulky nodal metastasis surrounding the celiac axis had a much poorer 


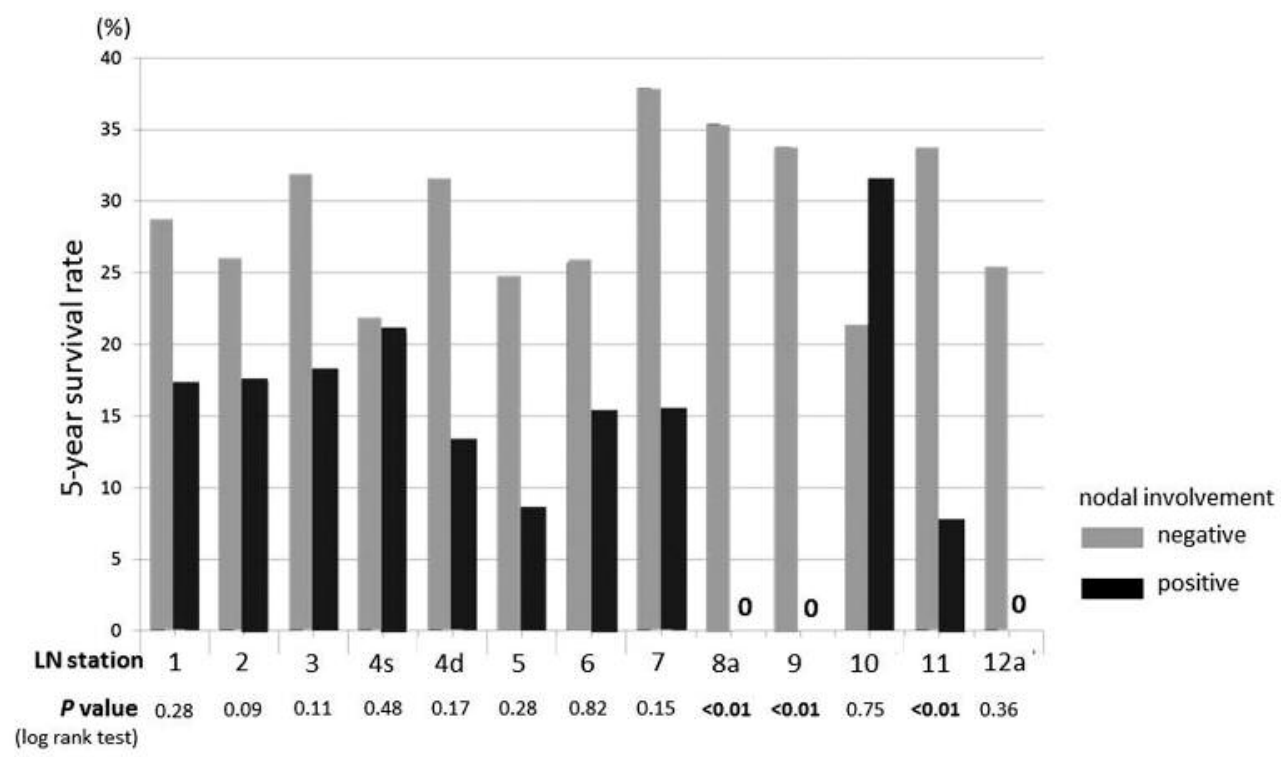

Figure 2. Overall survival of patients with or without metastasis at each regional nodal station. Gray lines are 5-year overall survival rates of patients without metastasis, while black lines are those with metastasis. All p-values shown under the graph are the analysis of log-rank test comparing the survival with or without each nodal involvement. LN, Lymph node.

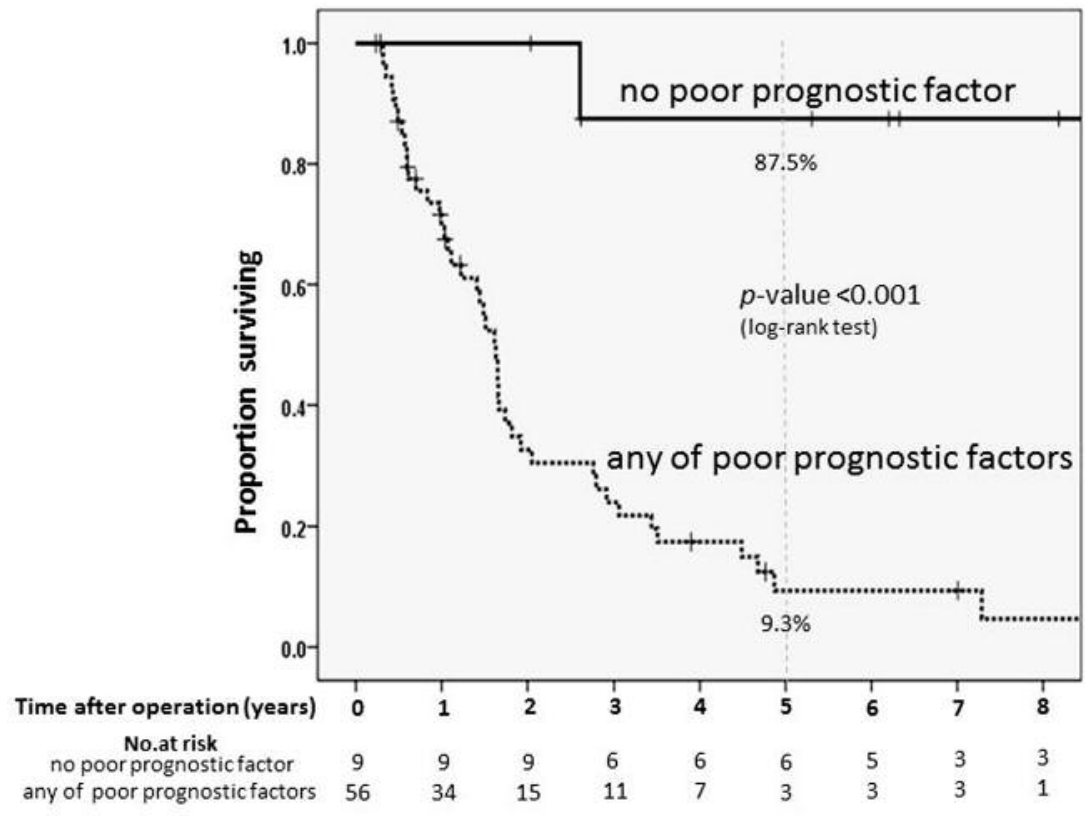

Figure 3. Kaplan-Meier curves of patients with or without poor prognostic factors. The survival curve of patients with pathologically-positive para-aortic lymph node without any of poor prognostic factors a solid line and that with any of poor prognostic factors is a dotted line. The identified poor prognostic factors are (i) tumor size $\geq 120 \mathrm{~mm}$, (ii) number of positive para-aortic lymph nodes $\geq 3$, (iii) nodal involvement at any of No. 8 a, 9 or 11 station.

prognosis than patients with only PALN metastasis (13). The lymphatic stream from the stomach to the para-aortic area is likely to be anatomically complex in many patients (22). However, it is speculated that continuous infiltration of cancer cells through the lymphatic chain extending from around the celiac axis to the para-aortic area signifies the systemic spread of cancer. Conversely, skip metastasis to the PALNs through some sort of pathway may have a higher 
probability of cure. The presence of lymph node metastases around the celiac axis may be confirmed during the surgery combined with intraoperative pathological examination using frozen section.

Finally, the tumor size was identified as a prognostic factor in the current study. Some previous investigators have also demonstrated a survival benefit of PALN dissection in patients with tumor diameters of 50 to $100 \mathrm{~mm}$, while PALN dissection did not improve the prognosis of patients with a tumor diameter of $>100 \mathrm{~mm}$ (23). This may be explained by the fact that a predominant recurrence pattern of a very large tumor with serosal exposure might be associated with peritoneal metastasis. Indeed, in the current study, no patients with a tumor diameter of $>120 \mathrm{~mm}$ survived for $>3$ years after surgery. Additionally, most such patients $(61.5 \%)$ had peritoneal recurrence (data not shown). Macroscopic type 4 tumors have been identified as poor prognostic factors in other reports (17), although this was not detected in the current study. This is probably because the tumor size had a greater impact than the macroscopic tumor type.

The 5-year OS rate in the current study $(21.0 \%)$ was higher than that in a previous report (13.8\%) (17). The reason for this is difficult to explain but differences in the patients' backgrounds are likely to have played a role. However, the survival rate in the current study was consistent with those in previous reports of systematic PALN dissection without perioperative chemotherapy $(4,6,8,12$, 20). Simple comparison of the current data with previous data is impossible because the current cohort included a certain proportion of patients who underwent incomplete PALN dissection after chemotherapy. It is true that multidisciplinary treatment (a combination of chemotherapy and surgery) improves the survival rate.

In the current study, the most common site of recurrence was the PALNs. In past reports, the predominant site of recurrence after prophylactic systematic PALN dissection was the peritoneum, followed by the lymph nodes and liver (8). In these reports, however, the actual pathologic examination findings proved that PALN metastasis did not occur in a substantial number of patients. In another study, the PALNs were reportedly the most common site of recurrence after radical gastrectomy without PALN dissection in patients with clinically positive PALNs who received neoadjuvant chemotherapy (24). In the present study, PALN recurrence was observed in association with both complete and incomplete PALN dissection. Interestingly, PALN recurrence was even observed at the already dissected site in 10 patients. These facts indicate that perfect locoregional control of the PALN area by surgery may be difficult even after systematic dissection by experienced surgeons. Whether surgeons should perform complete or incomplete PALN dissection after a patient has responded to chemotherapy remains unclear and, thus, a randomized study to answer this question would be unreasonable. Given the
Table II. Univariate analysis of survival: Kaplan-Meier analysis of overall survival for the patient with pathologically-positive para-aortic lymph node.

\begin{tabular}{|c|c|c|c|c|}
\hline Variables & $\begin{array}{l}\text { No. of } \\
\text { patients }\end{array}$ & $\begin{array}{c}\text { 3-year } \\
\text { OS rate } \\
(\%)\end{array}$ & $\begin{array}{c}\text { 5-year } \\
\text { OS rate } \\
(\%)\end{array}$ & $\begin{array}{c}p \text {-Value } \\
\text { (log-rank) }\end{array}$ \\
\hline \multicolumn{5}{|l|}{ Age (years) } \\
\hline$<65$ & 43 & 33.6 & 20.2 & \\
\hline$\geq 65$ & 22 & 33.8 & 23.2 & 0.936 \\
\hline \multicolumn{5}{|l|}{ Macroscopic type } \\
\hline non-type 4 & 53 & 39.8 & 26.9 & \\
\hline type 4 & 12 & 9.2 & 0 & 0.025 \\
\hline \multicolumn{5}{|l|}{ Tumor diameter (mm) } \\
\hline$<120$ & 52 & 42.7 & 26.8 & \\
\hline$\geq 120$ & 13 & 0 & 0 & $<0.001$ \\
\hline \multicolumn{5}{|l|}{ Tumor location } \\
\hline Upper & 29 & 35.1 & 21.9 & \\
\hline Middle, Lower & 36 & 32.4 & 20.3 & 0.797 \\
\hline \multicolumn{5}{|l|}{ Histology } \\
\hline Differentiated & 16 & 50.5 & 34.6 & \\
\hline Undifferentiated & 49 & 28.1 & 16.4 & 0.079 \\
\hline \multicolumn{5}{|l|}{ Operation procedure } \\
\hline Distal gastrectomy & 17 & 30.6 & 11.5 & \\
\hline Total gastrectomy & 48 & 34.9 & 23.9 & 0.408 \\
\hline \multicolumn{5}{|l|}{$\begin{array}{l}\text { PALN metastasis } \\
\text { during surgery }\end{array}$} \\
\hline No & 40 & 32.5 & 21.7 & \\
\hline Yes & 25 & 36.5 & 20.9 & 0.668 \\
\hline \multicolumn{5}{|l|}{ PAN dissection } \\
\hline & 29 & 28.9 & 13.8 & $\begin{array}{c}0.544 \\
\text { (sampling } \\
v s . \mathrm{b} 1 \text { ) }\end{array}$ \\
\hline b1dissection & 4 & 37.5 & 37.5 & $\begin{array}{c}0.698 \\
\text { (b1 vs. a2,b1) }\end{array}$ \\
\hline $\mathrm{a} 2, \mathrm{~b} 1$ dissection & 32 & 37.9 & 25.2 & $\begin{array}{c}0.405 \\
\text { (a2,b1 vs. } \\
\text { sampling) }\end{array}$ \\
\hline \multicolumn{5}{|c|}{ No. of total positive nodes } \\
\hline$<10$ & 10 & 75 & 62.5 & \\
\hline$\geq 10$ & 55 & 26.4 & 13.7 & 0.003 \\
\hline \multicolumn{5}{|l|}{ No. of positive PALN } \\
\hline$<3$ & 34 & 42.2 & 30.2 & \\
\hline$\geq 3$ & 31 & 23.8 & 10.6 & 0.004 \\
\hline \multicolumn{5}{|c|}{ Site of PALN involvement } \\
\hline $\mathrm{a} 2$ or $\mathrm{b} 1$ & 40 & 43.5 & 27.9 & \\
\hline both (a2 and b1) & 25 & 16 & 8 & 0.005 \\
\hline \multicolumn{5}{|c|}{ Pathological tumor depth } \\
\hline pT1-3 & 17 & 43.7 & 27.3 & \\
\hline pT4 & 48 & 30.1 & 19.1 & 0.27 \\
\hline \multicolumn{5}{|l|}{ Lymphatic invasion } \\
\hline ly $0-2$ & 39 & 42.4 & 28.7 & \\
\hline ly 3 & 26 & 20.3 & 10.2 & 0.161 \\
\hline \multicolumn{5}{|l|}{$\begin{array}{l}\text { Neoadjuvant } \\
\text { chemotherapy }\end{array}$} \\
\hline Presence & 55 & 37.7 & 22.7 & \\
\hline Absence & 10 & 15 & 15 & 0.092 \\
\hline \multicolumn{5}{|l|}{ Adjuvant chemotherapy } \\
\hline Presence & 42 & 38.3 & 25.5 & \\
\hline Absence & 23 & 27.2 & 13.6 & 0.756 \\
\hline
\end{tabular}

PALN, Para-aortic lymph node; OS, overall survival. 
Table III. Multivariate analysis of survival: Cox regression analysis of the prognostic factors for patients with pathologically positive paraaortic lymph node.

\begin{tabular}{lcccc}
\hline Variables & $\begin{array}{c}\text { No. of } \\
\text { patients }\end{array}$ & HR & $95 \%$ CI & $p$-Value \\
\hline $\begin{array}{l}\text { No. of positive PALN } \\
\quad 3\end{array}$ & 34 & & 1 & \\
$\quad \geq 3$ & 31 & 2.242 & $1.206-4.149$ & 0.011 \\
$\quad$ No. of positive nodes & & & & \\
$\quad<10$ & 10 & & 1 & \\
$\quad \geq 10$ & 55 & 2.874 & $0.852-9.687$ & 0.089 \\
$\begin{array}{l}\text { Macroscopic type } \\
\text { non type }\end{array}$ & 53 & & & \\
$\quad$ type 4 & 12 & 0.661 & $0.333-1.870$ & 0.435 \\
$\begin{array}{l}\text { Tumor diameter (mm) } \\
\quad<120\end{array}$ & 52 & & & \\
$\quad \geq 120$ & 13 & 3.372 & $1.181-9.625$ & 0.023 \\
$\begin{array}{l}\text { Nodal involvement } \\
\text { of No.8a or } 9 \text { or } 11\end{array}$ & & & & \\
$\quad$ negative & 15 & & 1 & \\
positive & 50 & 4.041 & $1.55-10.532$ & 0.004 \\
\hline
\end{tabular}

PALN, Para-aortic lymph node; OS, overall survival; CI, confidence interval; HR, hazard ratio.

outcomes of the current study, it seems realistic to perform limited PALN dissection when metastasis is suspected. In terms of surgical safety, acceptable outcomes have been revealed in previous studies when the surgery is performed by experienced surgeons $(10,13)$. Notably, however, fibrosis or edema of the tissue after a patient has responded to chemotherapy sometimes makes dissection much more difficult.

Our study has several limitations. First, this was a singleinstitution, retrospective study with a small sample size and selection bias. Second, the study period was long ( $>20$ years); during this time, the surgical staff, chemotherapy regimen and surgical quality/methods likely changed. The diagnostic imaging quality was also refined throughout the study period, although this study focused on patients with pathologicallypositive PALNs. Third, prognostic factors were estimated (including postoperative information, such as pathological findings); therefore, the optimal indication for PALN dissection cannot be predicted with total accuracy based on the present results. Ideally, prospective studies focusing on this topic should be conducted. However, considering the rarity of this disease situation, randomized studies seem unrealistic. The aforementioned FLOT 5 study will likely provide some insight.

In conclusion, the results of this study have confirmed that the small population of patients with pathologically-positive PALNs actually survives for a long period of time; however, the indications for PALN dissection should be carefully considered. Large tumors, nodal involvement around the
Table IV. The initial site of recurrence of the patient with PALN involvement.

\begin{tabular}{lc}
\hline The initial site of recurrence & No. of patients \\
\hline Lymph node & 24 \\
locoregional lymph nodes & 8 \\
PALN & 15 \\
(a2) & $(9)$ \\
(b1) & $(12)$ \\
Peritoneal & 15 \\
Hematogenious & 9 \\
Bone & 3 \\
Pleural & 2 \\
\hline
\end{tabular}

PALN, Para-aortic lymph node.

celiac axis and more than two involved PALNs indicate an unfavorable prognosis for patients with PALN metastasis.

\section{Conflicts of Interest}

The Authors declare that they have no conflicts of interest.

\section{Funding}

None.

\section{References}

1 Sobin LH: TNM classification of malignant tumors, 7th ed. 2009. UICC.

2 Japanese classification of gastric carcinoma: 3rd English edition. Gastric Cancer 14(2): 101-112, 2011.

3 Japanese Gastric Cancer Association. Japanese gastric cancer treatment guidelines (ver.4). 2014 (Tokyo: Kanehara publication).

4 Isozaki H, Okajima K, Fujii K, Nomura E, Izumi N, Mabuchi $\mathrm{H}$, Nakamura $\mathrm{M}$ and Hara $\mathrm{H}$ : Effectiveness of paraaortic lymph node dissection for advanced gastric cancer. Hepatogastroenterology 46(25): 549-554, 1999.

5 Baba M, Hokita S, Natsugoe S, Miyazono T, Shimada M, Nakano S, Takao S and Aikou T: Paraaortic lymphadenectomy in patients with advanced carcinoma of the upper-third of the stomach. Hepatogastroenterology 47(33): 893-896, 2000.

6 Sasako M, Sano T, Yamamoto S, Kurokawa Y, Nashimoto A, Kurita A, Hiratsuka M, Tsujinaka T, Kinoshita T, Arai K, Yamamura Y and Okajima K: D2 lymphadenectomy alone or with para-aortic nodal dissection for gastric cancer. N Engl J Med 359(5): 453-462, 2008.

7 de Manzoni G, Di Leo A, Roviello F, Marrelli D, Giacopuzzi S, Minicozzi AM and Verlato G: Tumor site and perigastric nodal status are the most important predictors of para-aortic nodal involvement in advanced gastric cancer. Ann Surg Oncol 18(8): 2273-2280, 2011.

8 Morita S, Fukagawa T, Fujiwara $\mathrm{H}$ and Katai $\mathrm{H}$ : The clinical significance of para-aortic nodal dissection for advanced gastric cancer. Eur J Surg Oncol 42(9): 1448-1454, 2016. 
9 Natsugoe S, Nakashima S, Matsumoto M, Nakajo A, Miyazono F, Kijima F, Ishigami S, Aridome K, Hokita S, Baba M, Takao $\mathrm{S}$ and Aikou T: Paraaortic lymph node micrometastasis and tumor cell microinvolvement in advanced gastric carcinoma. Gastric Cancer 2(3): 179-185, 1999.

10 Sano T, Sasako M, Yamamoto S, Nashimoto A, Kurita A, Hiratsuka M, Tsujinaka T, Kinoshita T, Arai K, Yamamura Y and Okajima K: Gastric cancer surgery: Morbidity and mortality results from a prospective randomized controlled trial comparing D2 and extended para-aortic lymphadenectomy - Japan Clinical Oncology Group study 9501. J Clin Oncol 22(14): 2767-2773, 2004.

11 Yonemura Y, Segawa M, Matsumoto H, Tsugawa K, Ninomiya I, Fonseca L, Fujimura T, Sugiyama K, Miwa K and Miyazaki I: Surgical results of performing R4 gastrectomy for gastric cancer located in the upper third of the stomach. Surg Today 24(6): 488-493, 1994.

12 Roviello F, Pedrazzani C, Marrelli D, Di Leo A, Caruso S, Giacopuzzi S, Corso G and de Manzoni G: Super-extended (D3) lymphadenectomy in advanced gastric cancer. Eur J Surg Oncol 36(5): 439-446, 2010.

13 Tsuburaya A, Mizusawa J, Tanaka Y, Fukushima N, Nashimoto A and Sasako M: Neoadjuvant chemotherapy with S-1 and cisplatin followed by D2 gastrectomy with para-aortic lymph node dissection for gastric cancer with extensive lymph node metastasis. Br J Surg 101(6): 653-660, 2014.

14 Wang Y, Yu YY, Li W, Feng Y, Hou J, Ji Y, Sun YH, Shen KT, Shen ZB, Qin XY and Liu TS: A phase II trial of Xeloda and oxaliplatin (XELOX) neo-adjuvant chemotherapy followed by surgery for advanced gastric cancer patients with para-aortic lymph node metastasis. Cancer Chemother Pharmacol 73(6): 1155-1161, 2014.

15 Park IH, Kim SY, Kim YW, Ryu KW, Lee JH, Lee JS, Park YI, Kim NK and Park SR: Clinical characteristics and treatment outcomes of gastric cancer patients with isolated para-aortic lymph node involvement. Cancer Chemother Pharmacol 67(1): 127-136, 2011.

16 Yoshida M, Ohtsu A, Boku N, Miyata Y, Shirao K, Shimada Y, Hyodo I, Koizumi W, Kurihara M, Yoshida S and Yamamoto S: Long-term survival and prognostic factors in patients with metastatic gastric cancers treated with chemotherapy in the Japan Clinical Oncology Group (JCOG) study. Jpn J Clin Oncol 34(11): 654-659, 2004
17 Tokunaga M, Ohyama S, Hiki N, Fukunaga T, Aikou S and Yamaguchi T: Can superextended lymph node dissection be justified for gastric cancer with pathologically positive paraaortic lymph nodes? Ann Surg Oncol 17(8): 2031-2036, 2010.

18 Sakuramoto S, Sasako M, Yamaguchi T, Kinoshita T, Fujii M, Nashimoto A, Furukawa H, Nakajima T, Ohashi Y, Imamura H, Higashino M, Yamamura Y, Kurita A, and Arai K: Adjuvant chemotherapy for gastric cancer with S-1, an oral fluoropyrimidine. N Engl J Med 357(18): 1810-1820, 2007.

19 Dindo D, Demartines N and Clavien PA: Classification of surgical complications: A new proposal with evaluation in a cohort of 6336 patients and results of a survey. Ann Surg 240(2): 205-213, 2004

20 Yonemura Y, Wu CC, Fukushima N, Honda I, Bandou E, Kawamura T, Kamata T, Kim BS, Matsuki N, Sawa T and Noh SH: Randomized clinical trial of D2 and extended paraaortic lymphadenectomy in patients with gastric cancer. Int J Clin Oncol 13(2): 132-137, 2008.

21 Kinoshita T, Kinoshita T, Saiura A, Esaki M, Sakamoto H and Yamanaka T: Multicentre analysis of long-term outcome after surgical resection for gastric cancer liver metastases. Br J Surg 102(1): 102-107, 2015.

22 Takashima $\mathrm{S}$ and Kosaka T: Results and controversial issues regarding a para-aortic lymph node dissection for advanced gastric cancer. Surg Today 35(6): 425-431, 2005.

23 Kunisaki C, Akiyama H, Nomura M, Matsuda G, Otsuka Y, Ono H, Nagahori Y, Hosoi H, Takahashi M, Kito F and Shimada H: Comparison of surgical results of D2 versus D3 gastrectomy (para-aortic lymph node dissection) for advanced gastric carcinoma: A multi-institutional study. Ann Surg Oncol 13(5): 659-667, 2006

24 Inoue K, Nakane Y, Kogire M, Fujitani K, Kimura Y, Imamura H, Tamura S, Okano S, Kwon AH, Kurokawa Y, Shimokawa T, Takiuchi H, Tsujinaka T and Furukawa H: Phase II trial of preoperative S-1 plus cisplatin followed by surgery for initially unresectable locally advanced gastric cancer. Eur J Surg Oncol 38(2): 143-149, 2012. 